\title{
Long-term efficacy and effectiveness of a behavioural and community-based exercise intervention (Urban Training) to increase physical activity in patients with COPD: a randomised controlled trial
}

\author{
Ane Arbillaga-Etxarri (1) 1,2,3,4 Elena Gimeno-Santos (1) ${ }^{1,2,3,5,6}$, \\ Anael Barberan-Garcia $\mathbb{1}^{5,6}$, Eva Balcells ${ }^{2,7,8}$, Marta Benet (1) ${ }^{1,2,3}$ ', Eulàlia Borrell ${ }^{9,10,11}$, \\ Nuria Celorrio ${ }^{12}$, Anna Delgado 1,2,3 Carme Jané $^{13}$, Alicia Marin ${ }^{8,14}$, \\ Carlos Martín-Cantera (10 10,13,15, Mónica Monteagudo (10) 10,15, Nuria Montellà 9,10,11, \\ Laura Muñoz ${ }^{16}$, Pilar Ortega ${ }^{17}$, Diego A. Rodríguez ${ }^{2,7,8}$, Robert Rodríguez-Roisin ${ }^{6}$, \\ Pere Simonet ${ }^{10,18,19}$, Pere Torán-Monserrat ${ }^{10,11}$, Jaume Torrent-Pallicer ${ }^{1,2,3}$, \\ Pere Vall-Casas ${ }^{20}$, Jordi Vilaró ${ }^{21}$ and Judith Garcia-Aymerich (1) 1,2,3
}

@ERSpublications

Urban Training in COPD increased physical activity after 12 months but not in self-reported nonadherent patients http://ow.ly/dc2C30lnAEs

Cite this article as: Arbillaga-Etxarri A, Gimeno-Santos E, Barberan-Garcia A, et al. Long-term efficacy and effectiveness of a behavioural and community-based exercise intervention (Urban Training) to increase physical activity in patients with COPD: a randomised controlled trial. Eur Respir J 2018; 52: 1800063 [https://doi.org/10.1183/13993003.00063-2018].

ABSTRACT There is a need to increase and maintain physical activity in patients with chronic obstructive pulmonary disease (COPD). We assessed 12-month efficacy and effectiveness of the Urban Training intervention on physical activity in COPD patients.

This randomised controlled trial (NCT01897298) allocated 407 COPD patients from primary and hospital settings $1: 1$ to usual care $(n=205)$ or Urban Training $(n=202)$. Urban Training consisted of a baseline motivational interview, advice to walk on urban trails designed for COPD patients in outdoor public spaces and other optional components for feedback, motivation, information and support (pedometer, calendar, physical activity brochure, website, phone text messages, walking groups and a phone number). The primary outcome was 12 -month change in steps day $^{-1}$ measured by accelerometer.

Efficacy analysis (with per-protocol analysis set, $n=233$ classified as adherent to the assigned

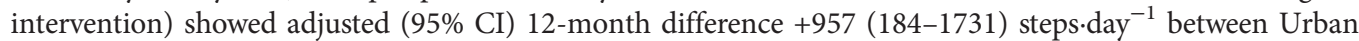
Training and usual care. Effectiveness analysis (with intention-to-treat analysis set, $\mathrm{n}=280$ patients completing the study at 12 months including unwilling and self-reported non-adherent patients) showed no differences between groups. Leg muscle pain during walks was more frequently reported in Urban Training than usual care, without differences in any of the other adverse events.

Urban Training, combining behavioural strategies with unsupervised outdoor walking, was efficacious in increasing physical activity after 12 months in COPD patients, with few safety concerns. However, it was ineffective in the full population including unwilling and self-reported non-adherent patients.

This article has supplementary material available from erj.ersjournals.com

Urban Training is trademark registered in Spain (ref. 3502702/9).

This study is registered at ClinicalTrials.gov with identifier number NCT01897298. The corresponding author can provide, upon request, individual participant data that underlie the results reported in this article (except variables, if any, that may allow identification of patients), after applying necessary measures to guarantee that no individual is identified or identifiable.

Received: Jan 112018 | Accepted after revision: Aug 062018

Copyright CERS 2018. This version is distributed under the terms of the Creative Commons Attribution NonCommercial Licence 4.0. 
Affiliations: ${ }^{1}$ ISGlobal, Barcelona, Spain. ${ }^{2}$ Pompeu Fabra University (UPF), Barcelona, Spain. ${ }^{3} \mathrm{CIBER}$ Epidemiología y Salud Pública (CIBERESP), Barcelona, Spain. ${ }^{4}$ Physical Activity and Sports Sciences, Faculty of Psychology and Education, University of Deusto, Donostia-San Sebastián, Spain. ${ }^{5}$ Respiratory Clinic Institute, Hospital Clinic of Barcelona, Barcelona, Spain. ${ }^{6}$ Institut d'Investigacions Biomèdiques August Pi i Sunyer (IDIBAPS)-Hospital Clínic, University of Barcelona, Barcelona, Spain. ${ }^{7}$ Pneumology Dept, Hospital del Mar, Institut Hospital del Mar d'Investigacions Mèdiques (IMIM), Barcelona, Spain. ${ }^{8} \mathrm{CIBER}$ Respiratory Diseases (CIBERES), Bunyola, Spain. 'Sant Roc Primary Healthcare Centre, Institut Català de la Salut (ICS), Badalona, Spain. ${ }^{10}$ Institut Universitari d'Investigació en Atenció Primària Jordi Gol (IDIAP Jordi Gol), Barcelona, Spain. ${ }^{11}$ Institute for Health Science Research Germans Trias i Pujol (IGTP), Badalona, Spain. ${ }^{12}$ Hospital de Viladecans, Viladecans, Spain. ${ }^{13}$ Passeig de Sant Joan Primary Healthcare Centre, Institut Català de la Salut (ICS), Barcelona, Spain. ${ }^{14}$ Pneumology Dept, Hospital Germans Trias i Pujol, Badalona, Spain. ${ }^{15}$ Universitat Autònoma de Barcelona, Bellaterra (Cerdanyola del Vallès), Spain. ${ }^{16}$ Agency for Health Quality and Assessment of Catalonia (AQuAS), Barcelona, Spain. ${ }^{17}$ Pneumology Dept, Hospital de Mataró, Mataró, Barcelona, Spain. ${ }^{18}$ Viladecans 2 Primary Healthcare Centre, Institut Català de la Salut (ICS), Viladecans, Spain. ${ }^{19}$ University of Barcelona, Barcelona, Spain. ${ }^{20}$ Universitat Internacional de Catalunya (UIC), Barcelona, Spain. ${ }^{21}$ FCS Blanquerna, Global Research on Wellbeing (GRoW), Ramon Llull University, Barcelona, Spain.

Correspondence: Judith Garcia-Aymerich, Barcelona Institute of Global Health (ISGlobal), Dr Aiguader 88, 08003 Barcelona, Spain. E-mail: judith.garciađisglobal.org

\section{Introduction}

Patients with chronic obstructive pulmonary disease (COPD) are substantially less active than their healthy peers [1] and this inactivity has been consistently related to a worse prognosis of the disease [2]. Thus, helping patients to adopt a more active lifestyle is a major goal in COPD management. Unfortunately, how to produce and maintain such behavioural change remains a challenge $[3,4]$.

Based on the beneficial effects of behavioural strategies on changing physical activity in patients with chronic diseases [5], recent COPD studies have focused on these kinds of interventions. Some of them, including physical activity counselling, pedometers or telecoaching (by computer or mobile technology) have reported increases in physical activity in the short term ( $\leqslant 4$ months) [6-8]. However, few studies followed patients for $\geqslant 1$ year $[6,9-11]$ and only one of them showed a sustained increase in physical activity, which was limited to a subset of patients [9]. Thus, one of the main difficulties of interventions to modify physical activity in COPD patients the achievement of a more prolonged long-term effect.

Given that currently available interventions are based mostly on patients' individual factors (biological and psychological), we argue that customising the interventions to patients' interpersonal (social support and cultural practices) and environmental (social, built and natural) determinants of physical activity [12] could help to maintain the increase in physical activity in the long term. Indeed, a report from the World Health Organization [13] suggests that interventions adapted to the local context and/or using existing social support and community structures are the most successful. In COPD, patients who live with others, walk the dog, take care of grandchildren or have an active partner have higher physical activity levels than those who do not, regardless of COPD severity and other individual characteristics [14-16], which suggests that interpersonal and environmental factors are key factors to include in future interventions.

Based on these premises we designed an intervention (Urban Training) consisting of motivational interviews, availability of outdoor walking trails specifically designed for exercise training of COPD patients [17] and other support components. We hypothesised that Urban Training could encourage COPD patients to increase and maintain their walking activity in the long term, because walking in public spaces is an extended cultural practice well integrated into the daily lifestyle of our COPD patients (elderly inhabitants of Mediterranean cities) [18].

We assessed the efficacy and effectiveness of the Urban Training intervention on physical activity level after 12 months of follow-up in patients with COPD. Secondary outcomes included severe COPD exacerbations, functional exercise capacity, body composition, health-related quality of life, anxiety and depression.

\section{Methods}

\section{Study patients}

Details on patient recruitment, randomisation and blinding are provided in online supplementary table S1. Briefly, we selected all subjects with a diagnosis of COPD according to the American Thoracic Society/ European Respiratory Society recommendations (post-bronchodilator forced expiratory volume in $1 \mathrm{~s}$ $(\mathrm{FEV} 1)$ to forced vital capacity $(\mathrm{FVC})$ ratio $<0.70)$ [19] who were seen in any of the participating 33 primary care and five hospital health centres from five Catalan seaside municipalities. We excluded patients with severe or life-threatening comorbidities, or those clinically unstable. The ethics committees of 
all participating institutions approved the study, along with the request for complete information exemption from patients, and all participants provided written informed consent.

\section{Study design and interventions}

This is a prospective, multicentre, parallel-group, randomised controlled trial registered at clinicaltrials.gov (NCT01897298) and reported according to the 2010 CONSORT statement [20] and its extension for non-pharmacological interventions [21]. Patients were allocated 1:1 to the Urban Training intervention or usual-care groups using random block sizes of six, eight and 10. The study consisted of four visits (figure 1): enrolment and baseline data collection; additional baseline data collection, randomisation and intervention 1 week later; 12-month data collection; and additional 12-month data collection 1 week thereafter.

Both groups received the usual standardised pharmacological and/or non-pharmacological treatment for COPD, including pulmonary rehabilitation, at the discretion of their physician and without any intervention by the research team.

Patients in the usual-care group were provided with general health counselling and the European Lung Foundation (ELF) information brochure "Living an active life with COPD" [22], which recommends $\geqslant 30$ min moderate physical activity $\geqslant 5$ days per week.

The Urban Training intervention consisted of the following six components (figure 2), detailed in the online supplementary material. 1) At baseline, a respiratory physiotherapist adequately trained in behavioural strategies used motivational interviewing techniques [23], integrated with a stage-matched approach [24], for a maximum of $1 \mathrm{~h}$. The interview was centred on empathy, reflective listening and affirmation, and addressed patients' resistance (personal difficulties, barriers and limitations) to eliciting behavioural change. Information on the remaining components of the intervention was provided during this interview. During the follow-up period, the physiotherapist administered up to four phone calls lasting 5-10 min to maintain motivation, depending on patients' self-efficacy and stage of change. 2) Participants received a dossier containing various maps of Urban Training walking trails, previously validated [17], according to their mobility options and preferences. Concisely, trails of different intensities (low, moderate or high, combining urban elements of varying intensity (stairs, ramps and types of surfacing)) were

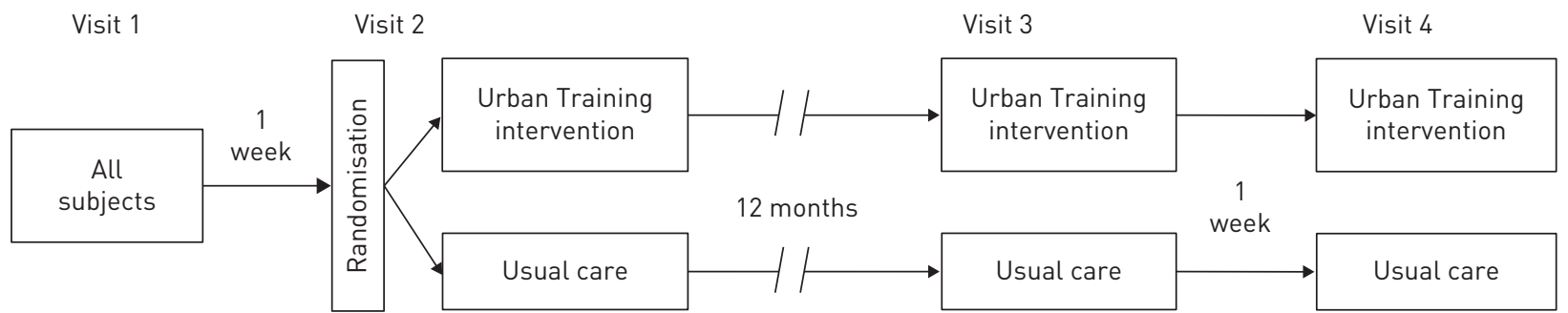

Informed consent
Baseline data collection (1):
- Sociodemographics
- Smoking status
- Dyspnoea
- Health-related quality of
- life
- Anxiety and depression
symptoms
- Cognitive impairment
- Exercise capacity
- Body composition
- Lung function
- Comorbidities
- Pharmacotherapy
- CoPD exacerbations
- Physical activity
laccelerometer deliveryl

Baseline data collection (2):

- Physical activity laccelerometer collection]

- Physical activity experience

Randomisation

Intervention administration 12-month data collection (1):

- Sociodemographics

- Smoking status

- Dyspnoea

- Health-related quality of life

- Anxiety and depression symptoms

- Cognitive impairment

- Exercise capacity

- Body composition

- Lung function

- Comorbidities

- Pharmacotherapy

- COPD exacerbations

- Physical activity

laccelerometer deliveryl 12-month data collection (2)

- Physical activity laccelerometer collection]

- Physical activity experience

- Satisfaction

- Adverse events 

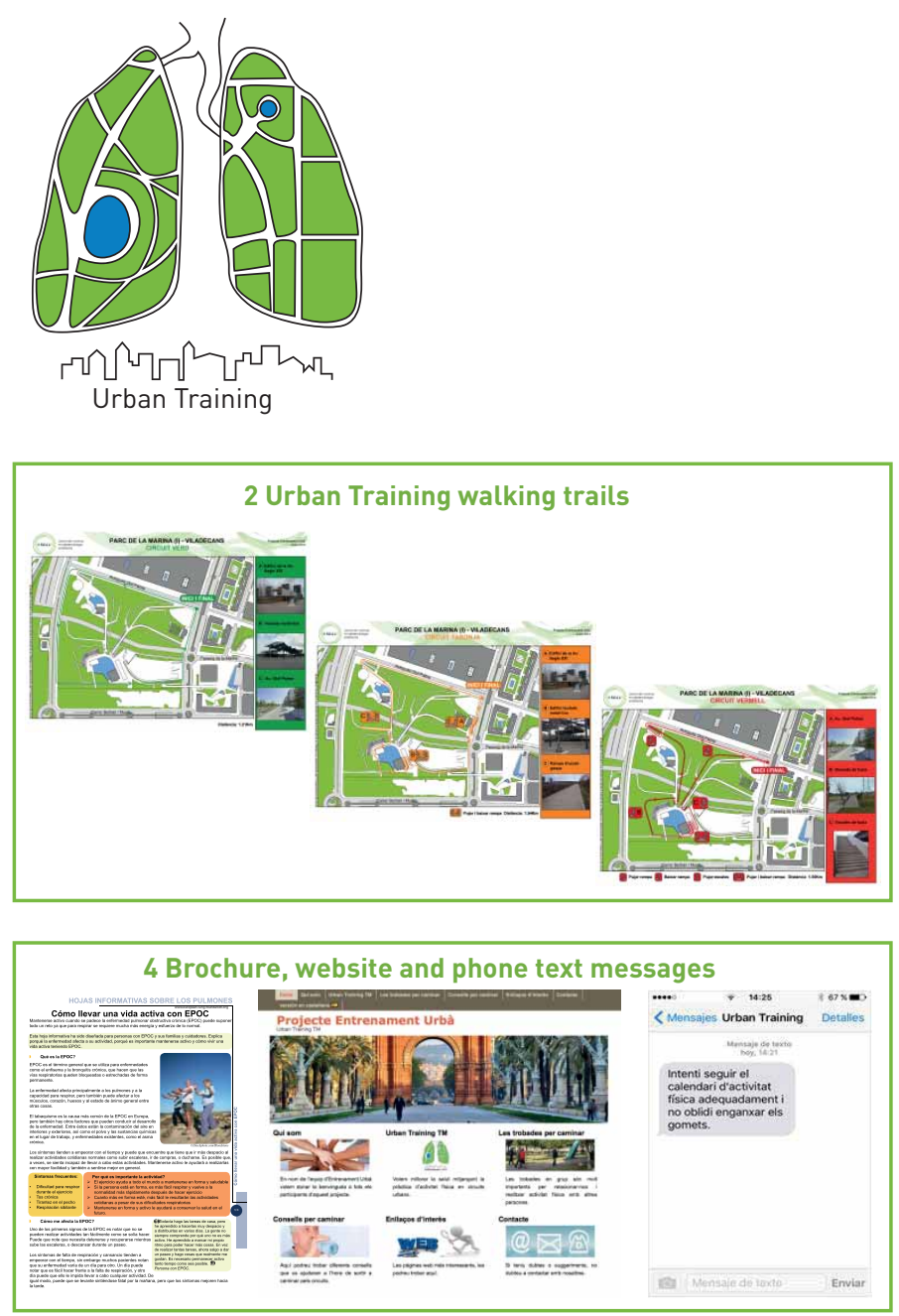
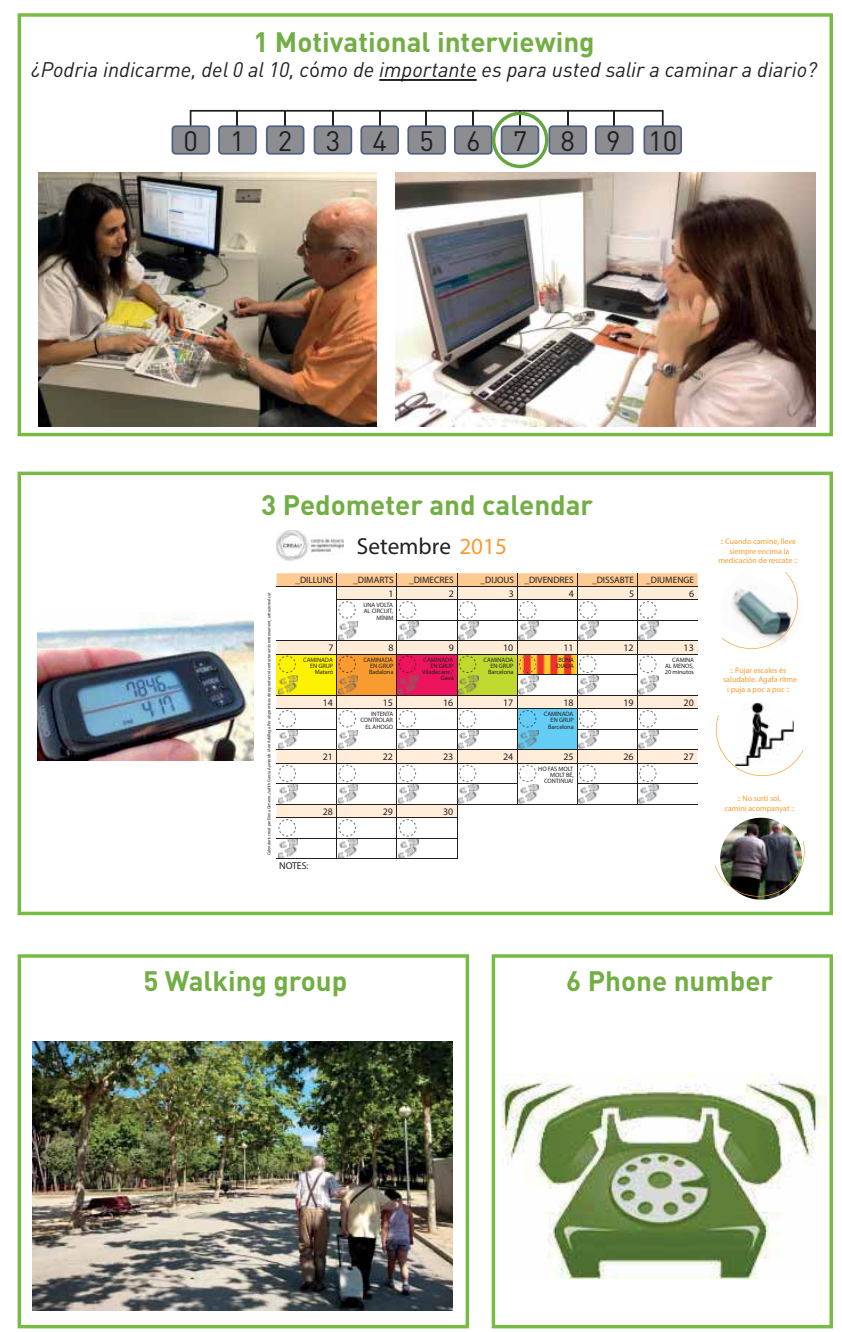

FIGURE 2 Components of the Urban Training intervention.

available in several walkable public spaces (boulevards, beaches and parks) of the five municipalities. The physiotherapist provided a complete explanation of trails characteristics and instructed patients to train following the FITT (frequency, intensity, time and type) principle [25]. Each patient was advised to start with a trail of intensity appropriate to his/her baseline dyspnoea and 6-min walking distance (6MWD), and instructed how to increase progressively the volume (number of walks per day on the same trail) and/ or the intensity of the trails during the following 12 months according to their symptoms and motivation (online supplementary figure S1). In all cases, the instructions were to walk at least one trail per day $\geqslant 5$ days per week, at a pace reaching a dyspnoea Borg scale score of 4-6 [26]. 3) Patients were provided with both a pedometer and a personalised calendar to monitor their physical activity and maintain motivation. 4) Patients received the same ELF information brochure as the usual-care group and the link to the project website (www.entrenament-urba.cat/). They were requested to provide a personal cell phone number where they would receive phone text messages every 2 weeks with educational or motivational messages. 5) Once per month during the follow-up period, patients could join a walking group for walking a trail accompanied by an experienced physical activity trainer. 6) Patients were given a phone number to contact the physiotherapists for any questions during follow-up. Of note, the Urban Training intervention was proposed as a supplement to the physical activities of daily life and in no case as a substitute activity.

\section{Procedures}

Full details and references on study procedures and quality control are available in the online supplementary material. Briefly, at baseline and 12 months we obtained the following data from all patients using standardised procedures. 1) Sociodemographic variables, smoking status, modified Medical Research Council dyspnoea scale, Clinical COPD Questionnaire (CCQ), COPD Assessment Test (CAT), Hospital Anxiety and Depression (HAD) scale and cognitive impairment (using phototesting) 
(interviewer-administered questionnaire); 2) 6-min walk test; 3) weight, height, body mass index (BMI) and fat-free mass index (FFMI) (physical examination and bioelectrical impedance); 4) FEV1 and FVC (pre- and post-bronchodilator spirometry); 5) comorbidities, pharmacological therapy and the number and severity of COPD exacerbations in the previous 12 months; 6) physical activity (Dynaport accelerometer; McRoberts BV, The Hague, The Netherlands), previously validated for COPD [27, 28]. A valid physical activity measurement was defined as $\geqslant 3$ days with $\geqslant 8 \mathrm{~h}$ of wearing time within waking hours [29]; compliance with the accelerometer was excellent (at baseline all patients fulfilled this criterion, median (range) wear was 7 (3-7) days, and recording time was 14.9 (11.1-15.0) h, of $15 \mathrm{~h}$ maximum from $07: 00 \mathrm{~h}$ to $22: 00 \mathrm{~h}$ ); at the final visit six $(2 \%)$ out of 286 patients did not fulfil the criterion of wearing time per day and, consequently, were excluded; among included patients, median (range) wear was 7 (4-7) days and recording time was $14.8(10.2-15) \mathrm{h}$; all patients included at least one weekend day both at baseline and final visit); and 7) physical activity experience (Clinical-PROactive Physical Activity (C-PPAC)). Additionally, at 12 months, patients answered a questionnaire about satisfaction with the study components and any potential adverse events experienced during or after walks in the previous 12 months. Finally, the physiotherapists administering both interventions noted down patients' spontaneous report of unwillingness to follow the instructions (e.g. walking $\geqslant 5$ days per week $\geqslant 30$ min.day ${ }^{-1}$ in the usual-care group or walking the Urban Training trails in the Urban Training group) at the baseline visit, as well as spontaneous reports of non-adherence (i.e. not having followed the instructions) at the 12-month visit.

\section{Study outcomes}

The primary outcome was the change in number of steps per day from baseline to 12-month follow-up. Secondary outcomes were having any severe COPD exacerbation (leading to hospital or emergency-room admission) during the 12-month follow-up and the 12-month changes in 6MWD, BMI, FFMI, CAT and CCQ total scores, and HAD-anxiety and -depression scores. Exploratory outcomes were the 12-month changes in phototest score, and total, amount and difficulty C-PPAC scores.

\section{Statistical analysis}

To detect a difference of 775 steps.day ${ }^{-1}$ (primary outcome) between groups (based on previous research about the effects of behavioural interventions in the elderly) [30], with a two-sided $\alpha=0.05$ and a power of $80 \%$, assuming a standard deviation of 3000 steps $\cdot$ day $^{-1}$ and a correlation between baseline and final steps $\geqslant 0.7$ (based on authors' data in COPD patients), a sample size of 142 patients per group was necessary. To account for a 30\% dropout rate during follow-up, we planned to recruit 202 participants per group (404 in total).

Prespecified efficacy and effectiveness were analysed using per-protocol and intention-to-treat (ITT) analysis sets, respectively. Briefly, ITT was defined as all randomised patients who completed the study at 12 months and provided a valid record of physical activity, while per-protocol was the subset of ITT who were classified as adherent to their corresponding intervention. Adherence was obtained from the interviews. We classified as "non-adherent" patients who 1) spontaneously reported at baseline that they were unwilling to follow any of the instructions; or 2) spontaneously reported at the 12-month visit that they had not been adherent to the study protocol (see the Procedures section). Remaining patients were labelled as "adherent". To test effectiveness, we built linear or logistic regression models, using the change from baseline to 12-month follow-up as the outcome, the intervention group as the main exposure variable and baseline levels of the corresponding outcome as a covariate (to account for individual differences in baseline levels). In efficacy analysis, we adjusted additionally for the variables related to adherence, since previous literature has shown that this adjustment may reduce the selection bias produced by a differential distribution of the reasons that moved participants to be adherent [31].

Post hoc analyses included stratification of efficacy results according to subgroups defined by baseline patient characteristics (online supplementary material). All analyses were redone using repeated measures ANOVA instead of linear regression. Safety analysis set included patients answering the adverse events questions at 12 months. All analyses were conducted with Stata 14.0 (StataCorp, College Station, TX, USA).

\section{Results}

Between 30 October 2013 and 29 January 2016, 552 stable COPD patients were assessed for eligibility and 407 patients underwent randomisation and received the corresponding intervention (figure 3, online supplementary table S2). 280 patients (69\% of the initial study population) completed the final visit and constituted the ITT analysis set (online supplementary table S3). These patients had higher physical activity and functional exercise capacity levels at baseline than those who did not participate in the final visit, both in the usual care and Urban Training group (online supplementary tables S3 and S4). Among followed patients, 233 patients ( $83 \%$ of the ITT) did not report unwillingness or non-adherence to the 


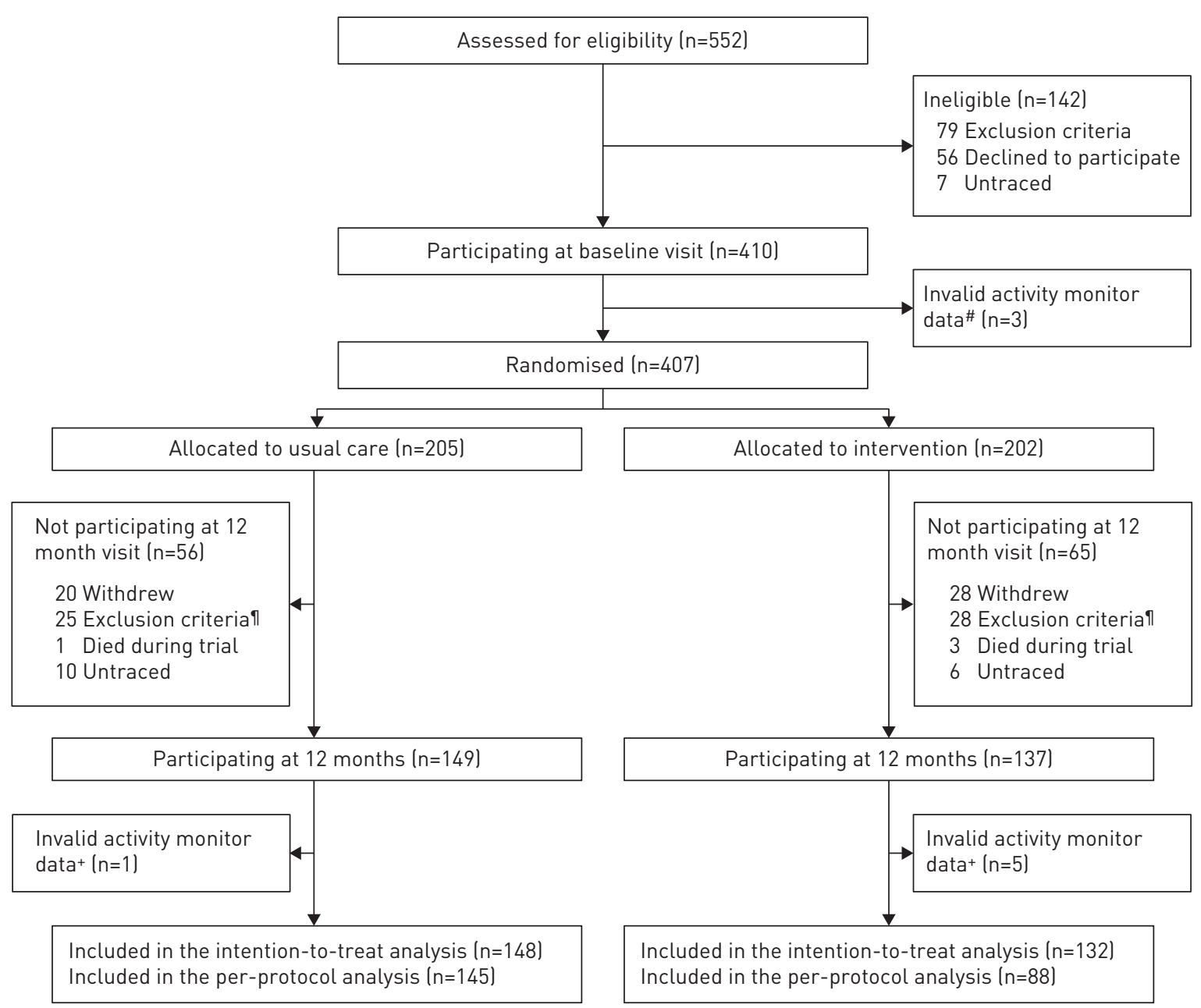

FIGURE 3 Flow of participants through the trial. \# : at baseline, three patients did not provide a valid record of physical activity due to technical reasons (e.g. patient entered the swimming pool and spoiled the record); ${ }^{\text {I: }}$ reasons for exclusion between baseline and 12 months were spending $>3$ months per year away from their home address $(n=7)$, mental disability $(n=3)$, severe comorbidity limiting survival at 1 year ( $=13)$ and another severe comorbidity $(n=30) ;{ }^{+}$: at the 12 -month visit, six $(2 \%)$ out of 286 patients did not fulfil the criterion of $\geqslant 3$ days with $\geqslant 8 \mathrm{~h}$ of wearing time within waking hours.

corresponding intervention and accordingly constituted the per-protocol analysis set. Patients who spontaneously reported unwillingness or non-adherence to the corresponding intervention had lower FEV1/FVC ratio, were most often current smokers, had diabetes in a higher proportion and showed higher values in the HAD-depression score than the rest of the patients (online supplementary table S5).

Baseline characteristics were similar in the per-protocol and ITT analysis sets and between two intervention groups (tables 1-3). Patients in the per-protocol analysis set were mostly male (88\%), mean $\pm \mathrm{SD}$ age $69 \pm 8$ years, had mild-to-very severe $\mathrm{COPD}$ ( $\mathrm{FEV} 158 \pm 17 \%$ predicted), preserved functional exercise capacity (6MWD $505 \pm 81 \mathrm{~m}$ ) and walked a mean \pm SD $8039 \pm 3964$ steps.day ${ }^{-1}$.

After 12 months, according to the per-protocol analysis set (efficacy analysis), patients in the usual-care group had not changed their physical activity, whereas those in the Urban Training group increased it by 816 steps $^{-d_{a y}}{ }^{-1}$ (figure 4 and table 2). In the analysis adjusted by factors independently related to adherence (FEV1/FVC ratio, smoking, diabetes and HAD-depression score; online supplementary table S6) and steps at baseline, the adjusted difference in steps between the Urban Training and usual-care groups was 957 (95\% CI 184-1731) steps.day ${ }^{-1}$ (figure 4 and table 2). There were no differences between intervention groups in any of the secondary outcomes or in cognitive impairment (exploratory outcome) (table 2). Positive changes (statistically significant better values) of physical activity experience were observed in the intervention group for the total, amount and difficulty scores. Stratification of efficacy results showed no significant differences between groups (figure 5). The adjusted difference at 12 months was $959(-72-1989)$ steps.day ${ }^{-1}$ for patients with mild-to-moderate COPD and $383(-860-1626)$ steps.day ${ }^{-1}$ for patients with severe-to-very severe COPD. Patients with higher physical activity levels at 
TABLE 1 Baseline characteristics of per-protocol and intention-to-treat analysis sets

\begin{tabular}{|c|c|c|c|c|c|c|}
\hline & \multicolumn{3}{|c|}{ Per-protocol analysis set } & \multicolumn{3}{|c|}{ ITT analysis set ${ }^{\#}$} \\
\hline & Usual care & $\begin{array}{l}\text { Urban } \\
\text { Training }\end{array}$ & All & Usual care & $\begin{array}{l}\text { Urban } \\
\text { Training }\end{array}$ & All \\
\hline Age years & $69 \pm 8$ & $69 \pm 9$ & $69 \pm 8$ & $69 \pm 8$ & $68 \pm 9$ & $69 \pm 8$ \\
\hline Female/male & $\begin{array}{c}17(12) / 128 \\
(88)\end{array}$ & $\begin{array}{c}12(14) / 76 \\
(86)\end{array}$ & $\begin{array}{c}29(12) / 204 \\
(88)\end{array}$ & $\begin{array}{l}18(12) / 130 \\
(88)\end{array}$ & $\begin{array}{c}18(14) / 114 \\
(86)\end{array}$ & $\begin{array}{c}36(13) / 244 \\
(87)\end{array}$ \\
\hline Active worker & $16(12)$ & $13(15)$ & $29(13)$ & $16(11)$ & $19(15)$ & $35(13)$ \\
\hline Dyspnoea mMRC grade (0-4) & $1 \pm 1$ & $1 \pm 1$ & $1 \pm 1$ & $1 \pm 1$ & $1 \pm 1$ & $1 \pm 1$ \\
\hline Post-bronchodilator FEV $1 \%$ pred & $58 \pm 18$ & $57 \pm 16$ & $58 \pm 17$ & $58 \pm 18$ & $56 \pm 17$ & $57 \pm 17$ \\
\hline Post-bronchodilator FEV1/FVC ratio & $0.55 \pm 0.12$ & $0.54 \pm 0.10$ & $0.54 \pm 0.12$ & $0.55 \pm 0.12$ & $0.53 \pm 0.11$ & $0.54 \pm 0.12$ \\
\hline $\begin{array}{l}\text { Airflow limitation }{ }^{+} \text {mild/moderate/severe/very } \\
\text { severe } \%\end{array}$ & $10 / 55 / 30 / 5$ & $8 / 57 / 31 / 4$ & $9 / 55 / 31 / 5$ & $10 / 54 / 30 / 6$ & $9 / 51 / 32 / 8$ & $10 / 53 / 31 / 6$ \\
\hline Charlson index & $2(1-3)$ & $1(1-2)$ & $2(1-3)$ & $2(1-3)$ & $2(1-3)$ & $2(1-3)$ \\
\hline Inhaled corticosteroids (alone or in combination) & $81(57)$ & $47(55)$ & $128(56)$ & $82(57)$ & $68(53)$ & $150(55)$ \\
\hline $\begin{array}{l}\text { Long-acting bronchodilators (LAMA or LABA, alone } \\
\text { or in combination) }\end{array}$ & $113(80)$ & $73(86)$ & $186(82)$ & $116(80)$ & 109 (85) & $225(82)$ \\
\hline Pulmonary rehabilitation at baseline & $6(4)$ & $5(6)$ & $11(5)$ & $6(4)$ & $6(5)$ & $12(4)$ \\
\hline Pulmonary rehabilitation during follow-up & $6(4)$ & $3(3)$ & $9(4)$ & $6(4)$ & $6(5)$ & $12(4)$ \\
\hline
\end{tabular}

Data are presented as $\mathrm{n}$, mean $\pm \mathrm{SD}, \mathrm{n}(\%)$ or median (interquartile range). ITT: intention-to-treat; mMRC: modified Medical Research Council; FEV1: forced expiratory volume in 1 s; FVC: forced vital capacity; GOLD: Global Initiative for Chronic Obstructive Lung Disease; LAMA: longacting muscarinic antagonist; LABA: long-acting $\beta$-agonist. \#: some variables have missing values, as follows. Per-protocol analysis set: socioeconomic status ( $n=1)$ active worker $(n=10)$, GOLD 2017 assessment $(n=2)$, cardiovascular disease, diabetes and musculoskeletal disease $(n=1)$, Charlson index $(n=1)$ and inhaled corticosteroids and long-acting bronchodilators ( $n=6)$; ITT analysis set: socioeconomic status ( $n=2)$, active worker ( $n=11$ ), GOLD 2017 assessment ( $n=3)$, cardiovascular disease, diabetes and musculoskeletal disease ( $n=1)$, Charlson index ( $n=1$ ) and inhaled corticosteroids and long-acting bronchodilators $(n=6)$; ๆ: UK National Statistics Socio-economic Classification III, IV or V; ${ }^{+}$: chronic obstructive pulmonary disease severity mild ( $F E V_{1} \geqslant 80 \%$ pred), moderate ( $F E V_{1} 50-79 \%$ pred), severe $F^{2} V_{1}$ ( $30-49 \%$ pred), very severe ( $F E V_{1}$ $<30 \%$ pred) and A (low risk, low symptom burden), B (low risk, high symptom burden), C (high risk, low symptom burden), D (high risk, high symptom burden); §: cardiovascular disease (International Classification of Diseases, 10th revision (ICD-10) I00-199), diabetes mellitus (ICD-10 E10-E14), musculoskeletal diseases (ICD-10 M00-M99).

baseline had higher increase during follow-up (adjusted difference in steps 1268 (158-2379) steps.day ${ }^{-1}$ versus $704(-429-1837)$ steps.day $\left.{ }^{-1}\right)$, although there was no sign of statistical interaction.

After 12 months, in the ITT analysis set (effectiveness analysis), there were no differences between intervention groups in any of the primary, secondary or exploratory outcomes (figure 4 and table 3). Analyses with repeated measures ANOVA provided very similar results.

Patients in the Urban Training group reported higher frequency of lower extremity muscle pain during walks than patients in the usual care group ( 38 versus $25 \%, \mathrm{p}=0.031$ ) without differences in any of the remaining adverse events (table 4).

Of the 132 patients of the intervention group participating in the follow-up visit, $70 \%, 87 \%$ and $90 \%$ used the trails maps, calendars and pedometers, respectively; $31 \%$ participated at least once in the walking groups; $41 \%$ contacted the researchers via phone during follow-up; and $2 \%$ visited the study website. At the 12 -month visit, $65 \%$ of patients delivered the calendars, and the mean \pm sD fulfilled months was 9 \pm 4 months. Satisfaction with the study and study staff was very high (mean satisfaction $\geqslant 9$ in a score ranging from 0 to 10) both in the usual-care and Urban Training groups (online supplementary table S7). Satisfaction with the study components in the Urban Training group was high or very high: $9.1 \pm 1.6$ for trail maps, $9.1 \pm 1.7$ for calendars, $9.0 \pm 1.8$ for pedometers, $7.5 \pm 2.8$ for walking groups, $9.4 \pm 1.0$ for phone text messages, $9.5 \pm 1.4$ for study phoneline and $8.7 \pm 2.3$ for study website (online supplementary table S7).

\section{Discussion}

This randomised controlled trial showed that the Urban Training intervention is more efficacious than usual care in increasing physical activity after 12 months in patients with COPD, with few safety concerns. 
TABLE 2 Efficacy results (per-protocol analysis set) of Urban Training intervention at 12 months in chronic obstructive pulmonary disease (COPD) patients

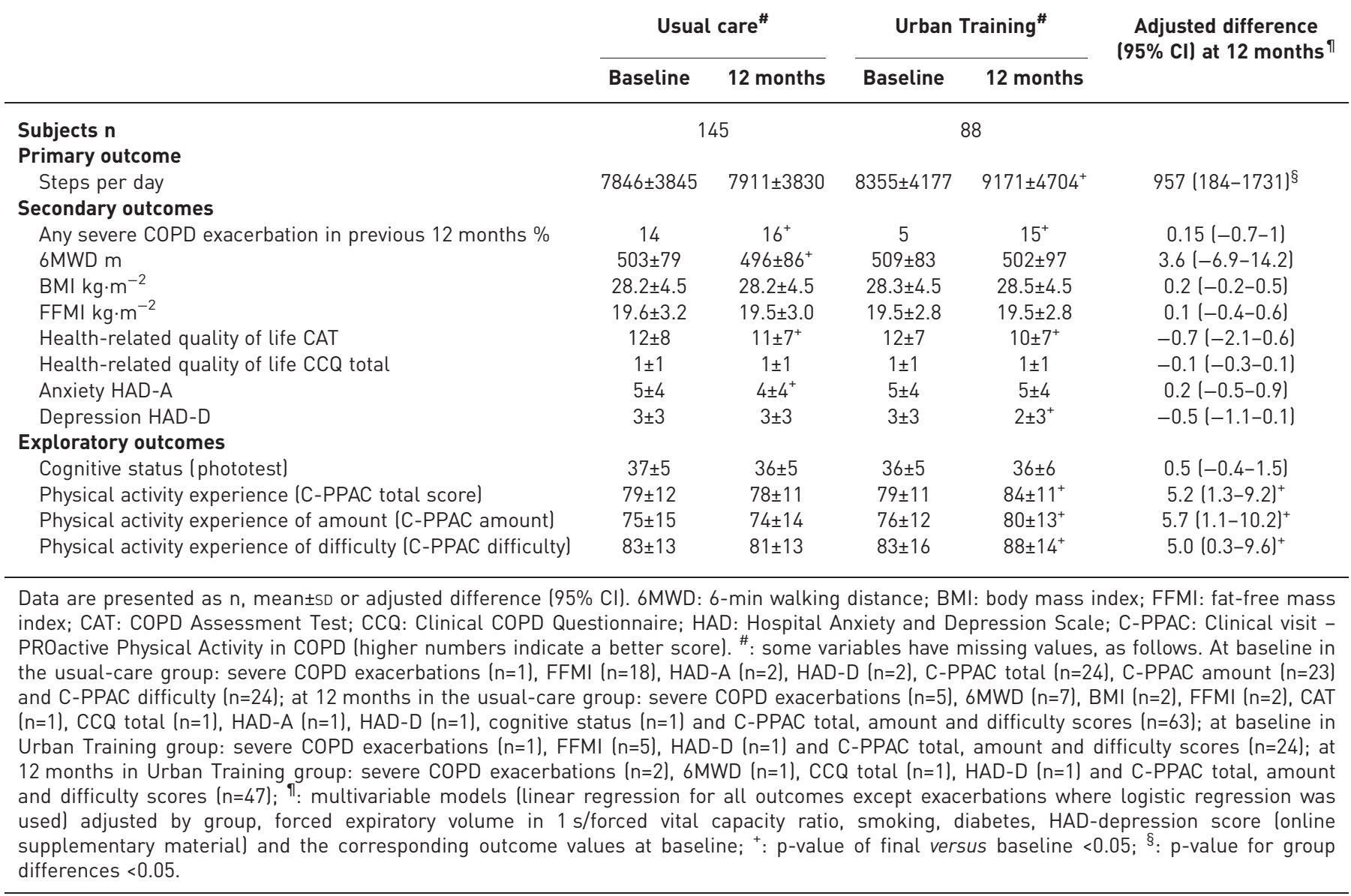

However, the intervention was not effective according to results with the ITT analysis set, suggesting that it improves physical activity only in willing, adherent patients. No effect of the intervention was found on severe COPD exacerbations, functional exercise capacity, body composition, health-related quality of life, anxiety or depression, in either analysis approach.

The main finding of this study is that the Urban Training intervention increased physical activity in COPD patients 1) at long-term (after 12 months) and 2) in a large scale of magnitude. Most studies testing the effects of behavioural physical activity interventions in COPD patients have successfully resulted in positive effects only at short-term ( $\approx 3$ months) $[6,7]$, and only one reported a long-term increase, which was restricted to a post hoc subgroup analysis [9]. Examination of the content of previous and current successful physical activity interventions allows us to hypothesise that the combination of motivational interviews, pedometers and diaries/calendars may be key for the long-term effect. The $\approx 900$ steps day $^{-1}$ increase observed in the Urban Training group lies within the defined limits of the minimal important difference in COPD patients (between 600 and 1100 steps.day $^{-1}$ ) [32] and is greater

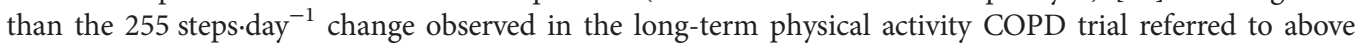
and the mean 808 steps.day ${ }^{-1}$ change identified in a review of pedometer-based physical activity interventions in older adults (including follow-ups between 2 weeks and 23 months) [30]. Our contention is that customising walking trails to patients' individual (e.g. exercise capacity and motivation), interpersonal (e.g. social support and cultural habit of walking) and environmental factors (e.g. lack of steep stairs in walking trails and home proximity or bus access to them) may have contributed to the long-term duration and large magnitude of the intervention effect. Therefore, Urban Training appears to be an attractive intervention potentially feasible due to its simplicity and reduced burden.

Potential harms of the Urban Training intervention need to be discussed. First, patients in the Urban Training group reported lower-extremity muscle pain in a higher proportion than patients in the 
TABLE 3 Effectiveness results (intention to treat analysis set) of Urban Training intervention at 12 months in chronic obstructive pulmonary disease (COPD) patients

\begin{tabular}{|c|c|c|c|c|c|}
\hline & \multicolumn{2}{|c|}{ Usual care ${ }^{\#}$} & \multicolumn{2}{|c|}{ Urban Training ${ }^{\#}$} & $\begin{array}{l}\text { Adjusted difference } \\
(95 \% \mathrm{CI}) \text { at } 12 \text { months }\end{array}$ \\
\hline \multicolumn{6}{|l|}{ Primary outcome } \\
\hline Steps per day & $7783 \pm 3847$ & $7825 \pm 3850$ & $8069 \pm 4554$ & $8002 \pm 4635$ & $-24(-741-693)$ \\
\hline \multicolumn{6}{|l|}{ Secondary outcomes } \\
\hline $\mathrm{BMI} \mathrm{kg} \cdot \mathrm{m}^{-2}$ & $28.3 \pm 4.6$ & $28.3 \pm 4.5$ & $28.4 \pm 5.0$ & $28.5 \pm 5.2$ & $0.0(-0.3-0.4)$ \\
\hline FFMI $\mathrm{kg} \cdot \mathrm{m}^{-2}$ & $19.6 \pm 3.2$ & $19.5 \pm 3.0$ & $19.6 \pm 3.0$ & $19.6 \pm 3.1$ & $0.1(-0.4-0.5)$ \\
\hline Health-related quality of life (CAT) & $12 \pm 8$ & $11 \pm 7$ & $12 \pm 7$ & $11 \pm 7^{+}$ & $0.1(-1.1-1.2)$ \\
\hline Health-related quality of life (CCQ total) & $1 \pm 1$ & $1 \pm 1$ & $1 \pm 1$ & $1 \pm 1^{+}$ & $-0.1(-0.3-0.1)$ \\
\hline Anxiety (HAD-A) & $5 \pm 4$ & $4 \pm 4^{+}$ & $5 \pm 4$ & $5 \pm 4^{+}$ & $0.2(-0.4-0.9)$ \\
\hline Depression (HAD-D) & $3 \pm 3$ & $3 \pm 3$ & $4 \pm 3$ & $3 \pm 3^{+}$ & $-0.5(-1.0-0.1)$ \\
\hline \multicolumn{6}{|c|}{ 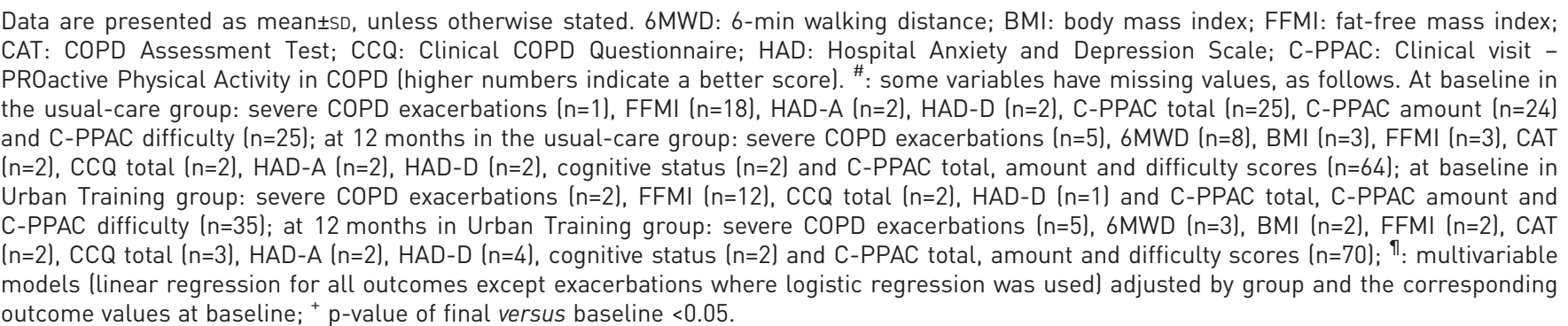 } \\
\hline
\end{tabular}

usual-care group, without differences in lower-extremity joint pain or other adverse events. This could be attributed to the fact that the Urban Training walking trails included ramps and stairs that may promote eccentric work of the leg muscles, which may result in muscle but not joint pain [33]. Second, although a recent trial has reported an acute increase in respiratory symptoms after walking in urban polluted areas [34], we did not collect information on these potential adverse events because 1) most of the trails were located in green or blue areas and 2) residential air pollution exposure was comparable between groups by design. Finally, the fact that patients included in the ITT but not in the per-protocol analysis set experienced greater decline in physical activity than those in the per-protocol analysis set could suggest that the intervention was harmful for them (which could have made them non-adherent). However, this is not supported by the fact that they experienced the same frequency of adverse events during or after walks as the rest of the Urban Training group and that a natural decline of physical activity levels has been observed previously in the absence of interventions $[35,36]$.

The Urban Training intervention did not improve most of the secondary and exploratory outcomes. The lack of effect on functional exercise capacity was unexpected, since, based on the physiological response generated when walking the trails during the validation study [17], we hypothesised that the intervention could produce effects similar to those of typical exercise training interventions. However, the lack of daily supervision when walking the trails may have hindered patients from regularly achieve a minimum training intensity (e.g. walking at a pace that generates dyspnoea or fatigue scores between 4 and 6 in the Borg scale). Indeed, a previous intervention that increased both physical activity and functional exercise capacity after 3 months had included close patient supervision via telecoaching [8]. The remaining secondary outcomes (severe COPD exacerbations, body composition, quality of life, anxiety or depression) were not primarily targeted by any of the Urban Training components and their improvement was expected only as a result of the expected increase in physical activity. Based on our results, it is tempting to speculate that the improvement in physical activity levels would need to be sustained for a period $>12$ months in order to result in measurable changes in the other health outcomes. Another explanation is 
a)

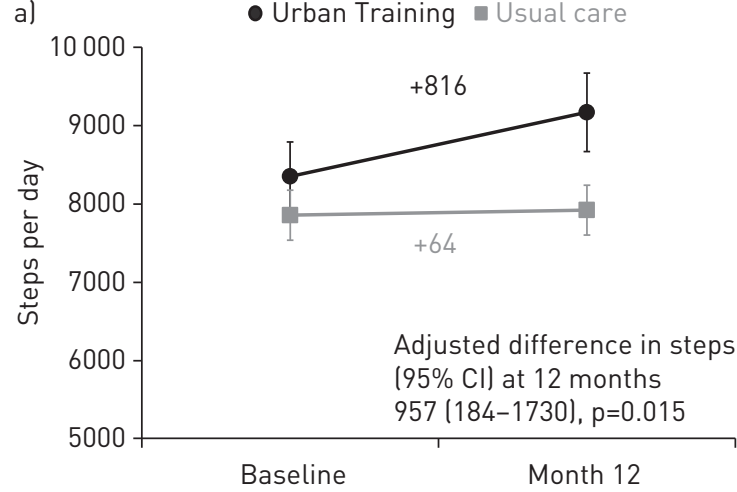

b)

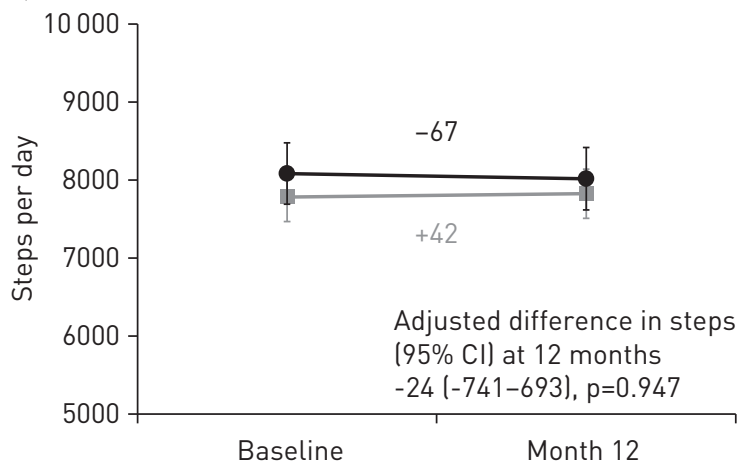

FIGURE 4 a) Efficacy and b) effectiveness results of Urban Training intervention on steps per day (primary outcome) at 12 months in chronic obstructive pulmonary disease patients. Data are presented as mean士SEM at baseline and 12 months.

that our patients already had a relatively good health status as per their values in COPD admissions, quality of life, anxiety or depression; therefore, they had little room for improvement. Finally, the Urban Training intervention improved patients' experience of their physical activity (exploratory outcome), in both the amount and difficulty dimensions, which supports that this concept provides complementary information to other related constructs such as health-related quality of life or exercise-induced symptoms [37].

The findings of this study are encouraging for COPD research and its management as well as for physical activity promotion in other populations. First, our findings highlight the consideration of patients' interpersonal (social and cultural) factors and environment when designing further interventions. From the clinical viewpoint, this approach may appear more feasible than others based strongly on technology solutions, particularly in countries with limited healthcare budgets. Second, our study supports the involvement of behaviour specialists in the design and administration of physical activity interventions or an equivalent acquisition of knowledge on behavioural techniques by health professionals who generally

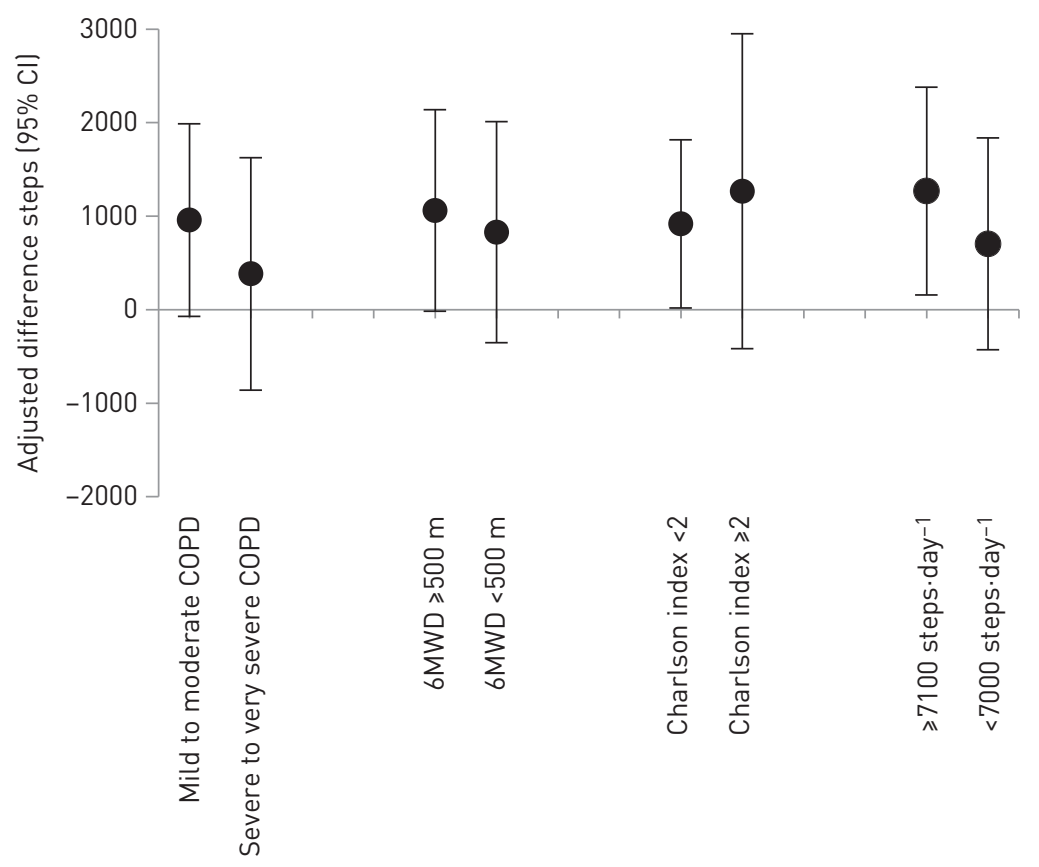

FIGURE 5 Efficacy of Urban Training intervention on steps per day (primary outcome) at 12 months in chronic obstructive pulmonary disease (COPD) patients according to subgroups based on baseline characteristics. Data are presented as adjusted difference $(95 \% \mathrm{Cl})$ at 12 months between intervention and usual-care groups. Subgroups defined by baseline airflow limitation stages (mild to moderate versus severe to very severe), functional exercise capacity (median 6-min walking distance (6MWD) $<500$ versus $\geqslant 500 \mathrm{~m}$ ), comorbidity (Charlson index $<2$ versus $\geqslant 2$ ) and physical activity levels (baseline $<7100$ versus $\geqslant 7100$ steps per day, cut-off equivalent to being adherent to physical activity recommendations for older adults) [30]. 


\begin{tabular}{|c|c|c|c|}
\hline & Usual care & Urban Training & p-value \\
\hline Subjects $\mathrm{n}$ & 142 & 128 & \\
\hline Any adverse event & 103 (73) & 99 (77) & 0.363 \\
\hline Lower-extremity joint pain & $38(27)$ & $41(32)$ & 0.342 \\
\hline Lower-extremity muscle pain & $36(25)$ & $48(38)$ & 0.031 \\
\hline General malaise or fatigue & $61(43)$ & $57(45)$ & 0.795 \\
\hline Dizziness & $12(8)$ & $9(7)$ & 0.821 \\
\hline Fainting & $1(1)$ & $0(0)$ & \\
\hline Dyspnoea & $48(34)$ & $46(36)$ & 0.713 \\
\hline Chest discomfort & $9(6)$ & $17(13)$ & 0.064 \\
\hline Palpitations & $22(16)$ & $23(18)$ & 0.586 \\
\hline Fall, twist or accident & $10(7)$ & $13(10)$ & 0.360 \\
\hline Cold, flu or pneumonia & $24(17)$ & $21(16)$ & 0.913 \\
\hline Heatstroke or dehydration & $1(1)$ & $2(2)$ & 0.605 \\
\hline
\end{tabular}

Data are presented as $\mathrm{n}(\%)$, unless otherwise stated.

exhibit a lack of training in behavioural change techniques [38, 39]. Finally, at the city level, interventions such as the Urban Training may contribute towards amortising the investment in public space (otherwise underused during certain times of the day) thus improving its sustainability. In fact, a close collaboration between health professionals and local governments has been promoted for example in the World Health Organization Healthy Cities project and is likely to result in social, economic and health benefits for all [40].

A limitation of the current study is that we defined adherence, and consequently the per-protocol analysis set, according to patient report. It is of note that we defined "non-adherence" from patient report and "adherence" otherwise. Thus, the ITT analysis set included, in the first place, patients who at baseline spontaneously reported unwillingness to undergo the intervention they had been assigned to. These patients are most often excluded from clinical trials, but we decided to keep them (and analyse their data) in order to provide effectiveness estimates. Second, the ITT analysis set included in addition patients who reported at the 12-month visit that they had not been adherent to the intervention to which they had been assigned, which in most cases, was due to a family situation (e.g. partner undergoing surgery). Again, some of these patients would be excluded in traditional clinical trials. Finally, the per-protocol analysis set included patients who did not make any spontaneous report in relation to their willingness or adherence, and probably comprised both adherent and non-adherent patients, thus underestimating the efficacy of Urban Training.

A second limitation is the apparent discrepancy between efficacy and effectiveness results. Of note, both approaches were prespecified in our analysis plan given previous reports in the literature about poor adherence to behavioural interventions $[9,41]$ and the well-known argument against ITT analysis (that it underestimates intervention effects in situations of non-adherence) [42]. The absence of effectiveness of Urban Training suggests the need for research to understand and eventually to identify ways to act upon the determinants of willingness and adherence to behavioural interventions in COPD. In our study, airflow limitation, smoking habits, diabetes and depression symptoms, but not physical activity levels were related to unwillingness or non-adherence, although collected information was not complete and there are no previous data on these issues to compare with. In addition, it has been disputed that the adherence to a given intervention may change dramatically after patients learn of trial findings, making the ITT effect estimation different from the effectiveness of the intervention in the community [43]. From a clinical viewpoint, patients who are willing to take an intervention such as Urban Training may be more interested in the per-protocol than the ITT effect.

Other shortcomings include the lack of intermediate assessments during the follow-up period, which could have given feedback to patients and would have allowed researchers to distinguish between short- and long-term effects. In addition, the fact that $\approx 30 \%$ of patients were lost to follow-up, a comparable figure to previous studies $[6,9,10]$, could have biased our results. Finally, our patients exhibited higher physical activity levels than those observed in previous studies [44-47], which could be considered a limitation of our research. However, a comparison of the clinical characteristics and physical activity levels of the patients included in the present and previous studies shows differences in physical activity both between countries (for the same severity of COPD) and within countries (for different severity stages and/or recruitment settings). We consider that, given that the Urban Training intervention was designed in a region characterised by relatively high social support, the cultural habit of walking, pedestrian accessibility 
to most outdoor public spaces, and a mild climate, it would be feasible in most Euro-Mediterranean cities. However, other geographic areas would need to conduct a proper adaptation to their social, cultural and environmental characteristics.

Strengths of the study are the novelty of customising the behavioural intervention to patients' interpersonal characteristics and environment, the large sample size and the measure of physical activity using an accelerometer. In addition, patients were recruited from primary care and hospitals of several municipalities, with barely any exclusion criteria, and diversity in relevant sociodemographic, lifestyle and clinical parameters, which make our results generalisable to a wide COPD population. The lack of differences in efficacy when patients were stratified according to their baseline features further supports the generalisability of our findings. With regard to the intervention, its simplicity and reduced burden make it possible to adapt it to other populations, including those with other chronic diseases and/or settings.

In conclusion, the Urban Training intervention, combining behavioural strategies with unsupervised outdoor walking, was efficacious in increasing physical activity after 12 months in COPD patients. However, it was ineffective in the full population including unwilling and self-reported non-adherent patients. The Urban Training intervention had no effect on severe COPD exacerbations, functional exercise capacity, body composition, health-related quality of life, anxiety or depression.

Acknowledgements: The authors thank all the technical staff of the Respiratory Diagnostic Centre from Hospital Clínic de Barcelona (Barcelona, Spain); Laura Gutierrez, Concepción Ballano, Anna Rodó-Pin, Bea Valeiro, Mireia Admetlló and Sergi Pascual from the Pneumology Department of Hospital del Mar (Barcelona); Alicia Francoso Vicente and Júlia Moraleda Hidalgo from the Pneumology Department of Hospital Germans Trias i Pujol (Badalona, Spain); and Marta Delicado and the Administration Department from the Viladecans 2 Primary care centre (Viladecans, Spain) for their contribution to the study.

Author contributions: A. Arbillaga-Etxarri and J. Garcia-Aymerich prepared the first draft of the paper; A. Arbillaga-Etxarri, M. Benet and J. Garcia-Aymerich had full access to the data and carried out statistical analysis. A. Arbillaga-Etxarri, E. Gimeno-Santos, A. Barberan-Garcia, E. Balcells, E. Borrell, N. Celorrio, A. Delgado, C. Jané, A. Marin, C. Martín-Cantera, M. Monteagudo, N. Montellà, P. Ortega, D.A. Rodríguez, P. Simonet, P. Torán-Monserrat, J. Torrent-Pallicer and J. Garcia-Aymerich contributed to data collection and coordination. All authors 1) provided substantial contributions to the conception or design of the work, or the acquisition, analysis or interpretation of data for the work; 2) revised the manuscript for important intellectual content; 3) approved the final version; and 4) agreed to be accountable for all aspects of the work. J. Garcia-Aymerich had full access to all of the data in the study and takes responsibility for the integrity of the data and the accuracy of the data analysis.

Conflict of interest: A. Arbillaga-Etxarri has nothing to disclose. E. Gimeno-Santos has nothing to disclose. A. Barberan-Garcia has nothing to disclose. E. Balcells has nothing to disclose. M. Benet has nothing to disclose. E. Borrell has nothing to disclose. N. Celorrio has nothing to disclose. A. Delgado has nothing to disclose. C. Jané has nothing to disclose. A. Marin has nothing to disclose. C. Martín-Cantera has nothing to disclose. M. Monteagudo has nothing to disclose. N. Montellà has nothing to disclose. L. Muñoz has nothing to disclose. P. Ortega has nothing to disclose. D.A. Rodríguez has nothing to disclose. R. Rodríguez-Roisin reports grants from Almirall and Menarini, personal fees for advisory board work from Boehringer Ingelheim, Pearl Therapeutics and TEVA, personal fees for lecturinf from Novartis and Takeda, during the conduct of the study, all related to COPD. P. Simonet reports personal fees for speaking from Menarini, Gebro, Teva, Boehringer, Rovi, AstraZeneca and GSK, outside the submitted work. P. Torán-Monserrat has nothing to disclose. J. Torrent-Pallicer has nothing to disclose. P. Vall-Casas has nothing to disclose. J. Vilaró has nothing to disclose. J. Garcia-Aymerich reports personal fees for consulting and lecture fees paid to institution from AstraZeneca, personal fees for lecturing from Esteve and Chiesi, outside the submitted work.

Support statement: The study was funded by grants from Fondo de Investigación Sanitaria, Instituto de Salud Carlos III (ISCIII, PI11/01283 and PI14/0419), integrated into Plan Estatal I+D+I 2013-2016 and co-funded by ISCIII-Subdirección General de Evaluación y Fomento de la Investigación and Fondo Europeo de Desarrollo Regional (FEDER); Sociedad Española de Neumología y Cirugía Torácica (SEPAR, 147/2011 and 201/2011), Societat Catalana de Pneumologia (Ajuts al millor projecte en fisioteràpia respiratòria 2013). ISGlobal is a member of the CERCA Programme, Generalitat de Catalunya. Anael Barberan-Garcia had personal funding from AGAUR 2014-SGR-661, Catalan Government. The funders had no role in study design, data collection and analysis, decision to publish, or preparation of the manuscript. Funding information for this article has been deposited with the Crossref Funder Registry.

\section{References}

1 Vorrink SN, Kort HS, Troosters T, et al. Level of daily physical activity in individuals with COPD compared with healthy controls. Respir Res 2011; 12: 33.

2 Gimeno-Santos E, Frei A, Steurer-Stey C, et al. Determinants and outcomes of physical activity in patients with COPD: a systematic review. Thorax 2014; 69: 731-739.

3 Leidy NK, Kimel M, Ajagbe L, et al. Designing trials of behavioral interventions to increase physical activity in patients with COPD: insights from the chronic disease literature. Respir Med 2014; 108: 472-481.

4 Singh S. One step at a time. Lifestyle physical activity interventions. Ann Am Thorac Soc 2016; 13: 586-587.

5 Conn VS, Hafdahl AR, Brown SA, et al. Meta-analysis of patient education interventions to increase physical activity among chronically ill adults. Patient Educ Couns 2008; 70: 157-172. 
6 Lahham A, McDonald CF, Holland AE. Exercise training alone or with the addition of activity counseling improves physical activity levels in COPD: a systematic review and meta-analysis of randomized controlled trials. Int J Chron Obstruct Pulmon Dis 2016; 11: 3121-3136.

7 Mantoani LC, Rubio N, McKinstry B, et al. Interventions to modify physical activity in patients with COPD: a systematic review. Eur Respir J 2016; 48: 69-81.

8 Demeyer $\mathrm{H}$, Louvaris $\mathrm{Z}$, Frei A, et al. Physical activity is increased by a 12-week semiautomated telecoaching programme in patients with COPD: a multicentre randomised controlled trial. Thorax 2017; 72: 415-423.

9 Altenburg WA, ten Hacken NHT, Bossenbroek L, et al. Short- and long-term effects of a physical activity counselling programme in COPD: a randomized controlled trial. Respir Med 2015; 109: 112-121.

10 Coultas DB, Jackson BE, Russo R, et al. A lifestyle physical activity intervention for patients with chronic obstructive pulmonary disease. A randomized controlled trial. Ann Am Thorac Soc 2016; 13: 617-626.

11 Moy ML, Martinez CH, Kadri R, et al. Long-term effects of an internet-mediated pedometer-based walking program for chronic obstructive pulmonary disease: randomized controlled trial. J Med Internet Res 2016; 18: e215.

12 Bauman AE, Reis RS, Sallis JF, et al. Correlates of physical activity: why are some people physically active and others not? Lancet 2012; 380: 258-271.

13 World Health Organization (WHO). Interventions on Diet and Physical Activity: What Works. Geneva, WHO, 2015.

14 Arbillaga-Etxarri A, Gimeno-Santos E, Barberan-Garcia A, et al. Socio-environmental correlates of physical activity in patients with chronic obstructive pulmonary disease (COPD). Thorax 2017; 72: 796-802.

15 Mesquita R, Nakken N, Janssen DJA, et al. Activity levels and exercise motivation in patients with COPD and their resident loved ones. Chest 2017; 151: 1028-1038.

16 Chen Z, Fan VS, Belza B, et al. Association between social support and self-care behaviors in adults with chronic obstructive pulmonary disease. Ann Am Thorac Soc 2017; 14: 1419-1427.

17 Arbillaga-Etxarri A, Torrent-Pallicer J, Gimeno-Santos E, et al. Validation of walking trails for the Urban Training of chronic obstructive pulmonary disease patients. PLoS One 2016; 11: e0146705.

18 Palacios-Ceña D, Alonso-Blanco C, Jiménez-Garcia R, et al. Time trends in leisure time physical activity and physical fitness in elderly people: 20 year follow-up of the Spanish population national health survey (1987-2006). BMC Public Health 2011; 11: 799.

19 Celli BR, MacNee W, Agusti A, et al. Standards for the diagnosis and treatment of patients with COPD: a summary of the ATS/ERS position paper. Eur Respir J 2004; 23: 932-946.

20 Hopewell S, Clarke M, Moher D, et al. CONSORT for reporting randomised trials in journal and conference abstracts. Lancet 2008; 371: 281-283.

21 Boutron I, Moher D, Altman DG, et al. Methods and processes of the CONSORT Group: example of an extension for trials assessing nonpharmacologic treatments. Ann Intern Med 2008; 148: W60-W66.

22 European Lung Foundation (ELF) 2013 Living an Active Life with COPD. Date last accessed: May 4 2016. Date last updated: February 2018. www.europeanlung.org/assets/files/en/publications/living-an-active-life-with-copd-en.pdf

23 William MR, Rollnick S. Motivational Interviewing: Preparing People for Change. New York, Guilford Press, 2002

24 Prochaska JO, Velicer WF. The transtheoretical model of health behavior change. Am J Health Promot 1997; 12: 38-48.

25 Pescatello L, Arena R, Riebe DTP. General principles of exercise prescription. In: ACSM's Guidelines for Exercise Testing and Prescription. 9th edn Philadelphia, Wolters Kluwer Health/Lippincott Williams \& Wilkins, 2013; pp. $166-177$.

26 Spruit MA, Singh SJ, Garvey C, et al. An official American Thoracic Society/European Respiratory Society statement: key concepts and advances in pulmonary rehabilitation. Am J Respir Crit Care Med 2013; 188: e13-e64.

27 Rabinovich RA, Louvaris Z, Raste Y, et al. Validity of physical activity monitors during daily life in patients with COPD. Eur Respir J 2013; 42: 1205-1215.

28 Van Remoortel H, Raste Y, Louvaris Z, et al. Validity of six activity monitors in chronic obstructive pulmonary disease: a comparison with indirect calorimetry. PLoS One 2012; 7: e39198.

29 Demeyer H, Burtin C, Van Remoortel H, et al. Standardizing the analysis of physical activity in patients with COPD following a pulmonary rehabilitation program. Chest 2014; 146: 318-327.

30 Tudor-Locke C, Craig CL, Aoyagi Y, et al. How many steps/day are enough? For older adults and special populations. Int J Behav Nutr Phys Act 2011; 8: 80.

31 Murray EJ, Hernán MA. Adherence adjustment in the Coronary Drug Project: a call for better per-protocol effect estimates in randomized trials. Clin Trials 2016; 13: 372-378.

32 Demeyer H, Burtin C, Hornikx M, et al. The minimal important difference in physical activity in patients with COPD. PLoS One 2016; 11: e0154587.

33 MacMillan NJ, Kapchinsky S, Konokhova Y, et al. Eccentric ergometer training promotes locomotor muscle strength but not mitochondrial adaptation in patients with severe chronic obstructive pulmonary disease. Front Physiol 2017; 8: 114.

34 Sinharay R, Gong J, Barratt B, et al. Respiratory and cardiovascular responses to walking down a traffic-polluted road compared with walking in a traffic-free area in participants aged 60 years and older with chronic lung or heart disease and age-matched healthy controls: a randomised, crossover study. Lancet 2018; 391: 339-349.

35 Waschki B, Kirsten AM, Holz O, et al. Disease progression and changes in physical activity in patients with chronic obstructive pulmonary disease. Am J Respir Crit Care Med.2015; 192: 295-306.

36 Clarenbach CF, Sievi NA, Haile SR, et al. Determinants of annual change in physical activity in COPD. Respirology 2017; 22: 1133-1139.

37 Gimeno-Santos E, Raste Y, Demeyer H, et al. The PROactive instruments to measure physical activity in patients with chronic obstructive pulmonary disease. Eur Respir J 2015; 46: 988-1000.

38 Blackmore C, Johnson-Warrington VL, Williams JE, et al. Development of a training program to support health care professionals to deliver the SPACE for COPD self-management program. Int J Chron Obstruct Pulmon Dis 2017; 12: 1669-1681.

39 Khan A, Dickens AP, Adab P, et al. Self-management behaviour and support among primary care COPD patients: cross-sectional analysis of data from the Birmingham Chronic Obstructive Pulmonary Disease Cohort. NPJ Prim Care Respir Med 2017; 27: 46. 
40 Rydin Y, Bleahu A, Davies M, et al. Shaping cities for health: complexity and the planning of urban environments in the 21st century. Lancet 2012; 379: 2079-2108.

41 Bourbeau J, Bartlett SJ. Patient adherence in COPD. Thorax 2008; 63: 831-838.

42 Hernán MA, Hernández-Díaz S. Beyond the intention-to-treat in comparative effectiveness research. Clin Trials 2012; 9: 48-55.

43 Hernán MA, Robins JM. Per-protocol analyses of pragmatic trials. N Engl J Med 2017; 377: 1391-1398.

44 Vorrink SNW, Kort HSM, Troosters T, et al. Efficacy of an mHealth intervention to stimulate physical activity in COPD patients after pulmonary rehabilitation. Eur Respir J 2016; 48: 1019-1029.

45 Burtin $\mathrm{C}$, Langer $\mathrm{D}$, van Remoortel $\mathrm{H}$, et al. Physical activity counselling during pulmonary rehabilitation in patients with COPD: a randomised controlled trial. PLoS One 2015; 10: e0144989.

46 Watz H, Waschki B, Boehme C, et al. Extrapulmonary effects of chronic obstructive pulmonary disease on physical activity: a cross-sectional study. Am J Respir Crit Care Med 2008; 177: 743-751.

47 Egan C, Deering BM, Blake C, et al. Short term and long term effects of pulmonary rehabilitation on physical activity in COPD. Respir Med 2012; 106: 1671-1679. 